\title{
Class I and Class III Antiarrhythmic Agents: Mechanisms of Action and the Problem of Proarrhythmic Activity
}

\author{
Vidal Essebag*
}

* To whom correspondence should be addressed: Faculty of Medicine, McGill University, Montreal, QC, Canada H3G 1 Y6

\section{INTRODUCTION}

Sudden cardiac death is a leading cause of mortality in industrialized nations, accounting for $50 \%$ of all cardiovascular deaths (1). Despite a decline in the past 20 years, sudden cardiac death accounts for 300,000 fatalities per year in the United States. Approximately $60 \%$ of these deaths occur in the absence of previously diagnosed heart disease (1). Nevertheless, in most instances of sudden cardiac death, the underlying pathophysiology of atherosclerotic cardiac disease with the superimposition of transient ischemic events makes the heart more susceptible to the onset and maintenance of lethal arrhythmias (1). Approximately 50\% of post-myocardial infarction (MI) fatalities are sudden cardiac deaths resulting mostly from ventricular tachycardia (VT) or ventricular fibrillation (VF). It is believed that these arrhythmias arise from mechanical dysfunction and ischemic events interacting within a disordered electrophysiologic milieu (2). This has prompted the active search for safe and effective treatment modalities and their ultimate evaluation in clinical trials. Currently, beta blockers (class II agents) are recommended for post-MI patients with frequent premature ventricular contractions (PVCs), whereas calcium antagonists (class IV agents) are not helpful, and class I agents are actually harmful (2). The benefit of class III drugs, particularly amiodarone, is presently being evaluated in large clinical trials. Therapeutic options for patients with sustained VT or VF occurring late after MI include pharmacologic antiarrhythmic therapy, transcatheter or surgical ablative therapy (for VT), and implantable cardioverter-defibrillators. Clinical trials have yet to determine which approach is most effective and under which circumstances (2). Treatment modalities for patients with nonsustained VT postMI are also being evaluated in clinical trials. This review specifically discusses the mechanisms of action of class I and class III antiarrhythmic agents as well as their potential untoward proarrhythmic effects.

\section{CLASS I ANTIARRHYTHMIC AGENTS}

The class I antiarrhythmic agents decrease automaticity in fast channel tissue by virtue of their $\mathrm{Na}^{+}$channel blocking property (which decreases the slope of phase 4 depolarization) The Class I drugs are furhter subdivided into three subclasses based on different effects on action potential duration (APD) and maximum velocity (Vmax); these effects result from varying potencies of $\mathrm{Na}^{+}$and $\mathrm{K}^{+}$channel block. (3). The class IA drugs, which include quinidine, disopyramide, and procainamide, reduce $\mathrm{Na}^{+}$channel availability by binding to the $\mathrm{Na}^{+}$channel in the open state (4), and reduce Vmax in both normal and diseased tissues. The class IA drugs can also increase APD (5). This effect may be related to their potent $\mathrm{K}^{+}$channel block during the plateau (phase 2) (6), which may be more significant than the $\mathrm{Na}^{+}$channel block, and therefore result in a 
prolongation of the plateau phase. The class IB drugs, which include lidocaine, tocainide, and mexiletine, are "pure" $\mathrm{Na}^{+}$channel blockers which do not block $\mathrm{K}^{+}$channels (2), and tend to decrease APD in the HisPurkinje system and in ventricular muscle. This decrease in APD is more significant in portions of the HisPurkinje system where the APD is normally longer (5). The class IB drugs, however, bind to the $\mathrm{Na}^{+}$channel largely in the inactivated (i.e., depolarized) state (4), and as a result, Vmax is selectively decreased in automatic ventricular tissue or in diseased tissues where the equilibrium potential is less negative (e.g., after myocardial infarction). The class IC drugs, which include flecainide, encainide, and propafenone, block $\mathrm{Na}^{+}$ channels in the open state (7) and also block $\mathrm{K}^{+}$channels (8), like the IA drugs. The difference is that the IC drugs block $\mathrm{Na}^{+}$channels more potently than the IA drugs such that IC drugs are more potent at reducing Vmax and in suppressing premature ventricular contractions. In addition, the class IC drugs cause a smaller increase in APD in ventricular muscle than do the IA, and actually decrease APD in the His-Purkinje system (5).

The use of class I drugs in antiarrhythmic therapy stems from their ability to decrease ventricular automaticity and thereby reduce ectopic beats which may trigger reentrant tachycardias. The effect of class I drugs in slowing conduction velocity in fast channel tissue has also been the basis for their use in the control of the rate of existing reentrant tachycardias. Despite these therapeutic effects, it is now known that the class I drugs also possess adverse effects which may outweigh the benefits of their antiarrhythmic effects. This point was clearly demonstrated in 1989 by findings of the Cardiac Arrhythmia Suppression Trial (CAST) (9) which revolutionized the use of antiarrhythmic drugs. CAST was designed to test the hypothesis that drugs with the ability to suppress ventricular premature depolarizations could decrease mortality in patients with asymptomatic or mildly symptomatic ventricular arrhythmias after myocardial infarction. However, the trial was discontinued when it was determined that the use of encainide or flecainide (two class IC drugs) actually increased the incidence of deaths from arrhythmias and non-fatal cardiac arrests (relative risk 3.6 with 95 percent confidence interval, 1.7 to 8.5 ) and also increased total mortality (relative risk 2.5 with 95 percent confidence interval, 1.6 to 4.5 ) when compared to patients on placebo (9). Therefore, it would appear that even though these drugs may be effective in suppressing an existing ventricular arrhythmia, they should not be used prophylactically in post-MI patients. The explanation for this increased mortality is that by potently slowing conduction, class IC drugs (and, to a lesser extent, the IA drugs) actually facilitate reentry and therefore increase the incidence of reentry tachycardias (10). Moreover, it appears that the magnitude of this proarrhythmic effect is directly related to the drugs' potency at slowing conduction (11).

\section{CLASS III ANTIARRHYTHMIC AGENTS}

As a result of the proarrhythmic effects of drugs which slow conduction velocity in ventricular tissue by blocking open $\mathrm{Na}^{+}$channels (i.e., Class IC and IA), a new emphasis has been made on searching for drugs which increase APD without affecting conduction by selectively blocking $\mathrm{K}^{+}$(and not $\mathrm{Na}^{+}$) channels in fast channel tissue. Drugs with such properties are classified as class III antiarrhythmic agents, and are all particularly effective in stopping the reentrant tachycardias in fast channel tissue. Reentry is stopped when the refractory period of myocardial fibers in the reentrant circuit is prolonged to such an extent that the propagating reentrant impulse no longer finds excitable myocardium but is blocked in refractory tissue (12). Hence, unlike the Class IC and IA drugs, the class III drugs do not increase the likelihood of developing reentrant tachyarrhythmias, but, on the contrary, make reentry more difficult. Nevertheless, we are now finding out that the class III drugs can also be proarrhythmic in their own way; by prolonging APD they can lead to a long QT syndrome where early afterdepolarizations (EADs) can trigger ventricular arrhythmias known as torsades de pointes (49). The earlier class III drugs (like sotalol and amiodarone) have properties in addition to increasing APD by blocking $\mathrm{K}^{+}$channels, while the newer class III agents (like sematilide WAY123,398, dofetilide, E-4031, ibutilide, and RP 58866) are more specific for prolonging refractoriness by selective $\mathrm{K}^{+}$channel blocking activity. Furthermore, even among the new class III agents, differences may 
exist in their selectivity for the different $\mathrm{K}^{+}$channel types.

\section{Early Class III Agents}

\section{Sotalol}

Sotalol is a class III antiarrhythmic drug which, in addition to increasing APD by blocking $\mathrm{K}^{+}$channels, also competitively antagonizes beta adrenergic receptors. This antiadrenergic activity can depress slow channel tissue by decreasing cAMP-dependent calcium entry. In doing so, these agents may prevent atrial premature beats which can act as

trigger to initiate atrial fibrillation. Sotalol is also effective at restoring sinus rhythm in patients with chronic atrial fibrillation (13), an effect largely due to its class III property of increasing atrial effective refractory period (14). Sotalol's adverse cardiovascular effects, which include atrioventricular block, bradycardia, hypotension, and exacerbation of heart failure, are mostly due to beta blockade (15). However, sotalol is a racemic mixture of d-and l- isomers, and while l-sotalol has beta-blocking effects comparable to d,l-sotalol, $\mathrm{d}$-sotalol is practically devoid of beta blocking properties but remains a potent class III agent (16). Therefore, $\mathrm{d}$-sotalol shares the APD prolonging properties of the racemic mixture without causing hypotension and bradycardia (17). D-sotalol has also been shown to decrease atrioventricular (AV) node automaticity by prolonging AV node APD, and has proven effective in abolishing AV nodal reentrant tachycardias by lengthening refractoriness in AV nodal cells (18). Sotalol has also been shown to significantly decrease the recurrence of arrhythmias, sudden death, and total cardiovascular mortality, when compared to various class I agents in the Electrophysiologic Versus Electrocardiographic Mortality (ESVEM) study (19). Sotalol's relative effectiveness is presumably related to its ability to prevent ventricular arrhythmias by increasing APD without the proarrhythmic effects of slowing conduction. However, since ESVEM did not use an independent control analogous to placebo, sotalol's effects cannot be interpreted in absolute terms.

Nevertheless, as Fitton and Sorkin concluded in a review on sotalol published in 1993, it was believed that sotalol was likely to prove particularly appropriate in the treatment and prophylaxis of life-threatening ventricular tachyarrhythmias (20). More recently, the role of d-sotalol in managing cardiac arrhythmias has been addressed in controlled clinical trials. However, the Survival With Oral D-sotalol (SWORD) doubleblind placebo-controlled trial in survivors of MI with depressed ventricular function was recently prematurely terminated because of a strikingly greater all-cause mortality compared with placebo (4.6 vs. $2.6 \%)(21,22)$. D-sotalol has also been shown to produce EADs and torsades de pointes in the rabbit heart $(23,24)$. These findings raise concerns regarding the current popular concept of using "pure" class III agents to control arrhythmias; these drugs' QT-prolonging effect may increase mortality precipitating EAD-induced fatal arrhythmias.

\section{Amiodarone}

Amiodarone is a unique class III drug; it possesses properties belonging to all four of the Singh and Vaughan Williams classes of antiarrhythmic agents. Analogous to lidocaine's class I property, amiodarone interacts selectively with the inactivated state of the $\mathrm{Na}^{+}$channel (25) and therefore acts preferentially in ischemic tissues. Amiodarone's $\mathrm{Na}^{+}$channel block, which reduces Vmax and slows ventricular conduction in a usedependent fashion, is largely responsible for its ability to slow ventricular tachycardias to a more hemodynamically-tolerated rate. Its high efficacy in suppressing premature ventricular complexes is believed to be largely due to its class I actions (25). Like sotalol, amiodarone also has antiadrenergic (class II) activity (26) which may contribute to its AV node suppressive actions. Amiodarone's Class III effects contribute to its ability to prevent atrial or ventricular reentrant arrhythmias (25). Amiodarone, like sotalol, prolongs the atrial effective refractory period (probably a class III property), and is more effective than sotalol in restoring and maintaining sinus rhythm in patients with chronic atrial fibrillation $(13,27)$. Amiodarone's calcium channel 
blocking (class IV) properties contribute to its ability to prevent AV node reentrant arrhythmias and to slow the ventricular response in atrial fibrillation. Furthermore, amiodarone's ability to block L-type calcium channels, which mediate EADs, may explain its apparent lack of precipitation of EAD-induced arrhythmias such as torsades de pointes, despite a prolonged QT interval (25). This fact, in addition to amiodarone's other actions, may have contributed to its success in three recent studies of secondary prevention in postmyocardial infarction patients - the Basel Antiarrhythmic Study of Infarct Survival (BASIS), the Polish Amiodarone Study, and a pilot study in Canada - where amiodarone succeeded in decreasing mortality (28). Amiodarone's favorable risk-to-benefit ratio is presently being examined in two large-scale clinical trials: the Canadian Amiodarone Myocardial Infarction Arrhythmia Trial (CAMIAT) and the European Myocardial Infarction Amiodarone Trial (EMIAT) (2). CAMIAT targets patients with frequent ventricular ectopic activity ( $>10 \mathrm{PVC} / \mathrm{hr}$ ) following MI, whereas EMIAT targets patients with poor LV function (left ventricular ejection fraction $<41 \%$ ) following MI (29).

\section{New Class III Agents}

Unlike sotalol and amiodarone, most of the new class III antiarrhythmic drugs mediate their effect via a single mode of action; they selectively prolong APD. Some of these new drugs do so by specifically blocking the delayed rectifier $\mathrm{K}^{+}$current (IK). Sematilide and WAY-123,398, for example, are class III drug that selectively prolongs APD by blocking IK $(30,31)$. As will be discussed, other new class III agents selectively block only the rapid activating component of the delayed rectifier current (IKr), while others act on other channel types.

\section{Dofetilide and E-4031}

Dofetilide (UK-68,798) and E-4031 are two new potent methanesulfonanilide class III agents which selectively inhibit the rapidly activating inward rectifying component of the net delayed rectifier $\mathrm{K}^{+}$current (IKr) $(32,33)$. These agents do so without effects on the larger but more slowly activating component (IKs) or on the inward rectifier $\mathrm{K}^{+}$current (IK1) $(34,35)$. Both dofetilide and E-4031 prolong APD in a "reverse" ratedependent manner (i.e., effects are greater at lower than at higher rates of stimulation). However, it was shown that the sensitivity to the block of IKr by dofetilide and E-4031 is rate-independent. Interestingly, the explanation for the reverse rate-dependence of APD prolongation results from the increase in magnitude of IKs at rapid rates as a result of incomplete deactivation of IKs (34). Another experiment demonstrated that isoproterenol (a beta adrenergic receptor agonist) increased the conductance of IKs channels without affecting IKr channels, and therefore decreases the APD prolonging effects of E-4031 (36). Therefore, the newly developed methanesulfonanilide drugs (dofetilide and E-4031) would be expected to have decreased efficacy in the presence of high sympathetic tone, high heart rates, or both (30). In addition to prolonging refractoriness, an experiment on dogs showed that methanesulfonanilide class III drugs may augment cardiac contractility (37). Other experiments on ferret ventricular papillary muscle also demonstrated that dofetilide and E-4031 increased contractility whereas d-sotalol had no effect on contractility (38).

\section{Other New Agents}

Ibutilide is a new class III drug which has been shown to be effective in converting sustained atrial fibrillation to sinus rhythm (39). It has also been shown to be effective in the prevention of atrial flutter in a canine model (40). Ibutilide's mode of action, however, differs from that of d-sotalol, sematilide, WAY-123,398, dofetilide, and E-4031. Rather than blocking an outward $\mathrm{K}^{+}$current, ibutilide prolongs APD largely through the activation of a slow inward $\mathrm{Na}^{+}$current (41) . In fact, at much higher concentrations, ibutilide actually increases an outward $\mathrm{K}^{+}$current (IK) (42). It was shown that a low concentration of ibutilide could prolong the APD beyond that already prolonged by one of the other class III drugs (presumably by increasing a late inward $\mathrm{Na}^{+}$current), while a high concentration of ibutilide did the opposite (presumably by increasing an 
outward $\mathrm{K}^{+}$current) (42). This dose-related effect on APD may be expected to help reduce the likelihood of torsades de pointes at high doses; however, most episodes of torsades are not dose related (43). Using a rabbit model, ibutilide was compared to d-sotalol, E-4031, and dofetilide, and was found to have a significantly lower incidence of EAD-induced torsades de pointes arrhythmias (ibutilide 12\%, d-sotalol 70\%, E-4031 56\%, dofetilide $69 \%$, saline $0 \%$ ) (44).

RP 58866 is another new class III drug with a unique mode of action; it acts by selectively blocking the inward rectifier current (IK1) (33) without having any effect on the delayed rectifier current (IK) (30).

The new agents NE-10064 (azimilide) and NE-10133 are reported to be potent at inhibiting the slowly activating potassium current (IKs) in guinea pig cardiac myocytes (45). Recently, however, NE-10064 was shown to block IKr more selectively than IKs, and to also block the L-type calcium current in a usedependent manner in guinea pig myocytes (46).

BRL-32872 is new class III agent reported to inhibit the rapidly activating component of the delayed rectifier potassium current and the L-type calcium current (believed to be responsible for causing EADs) in the guinea pig heart (47). BRL-32872 was also shown to lack a reverse frequency-dependent effect on APD, to rarely produce EADs, and to antagonize EADs produced by E-4031 (47). In another study using a minipig model, BRL-32872 demonstrated an antifibrillatory effect associated with prolonged ventricular repolarization and showed enhanced efficacy over dofetilide on reperfusion arrhythmias, presumably due to its calcium channel blocking property (48).

\section{CONCLUSION}

In summary, current class I antiarrhythmic agents are not suitable for prophylactic use because they promote reentrant tachycardias by slowing conduction velocity. Current class III drugs do not slow conduction velocity, but are nevertheless proarrhythmic. Their QT interval lengthening effect leads to EADs which cause arrhythmias such as torsades de pointes. Furthermore, some of the newest class III agents like dofetilide and E-4031 have the problem of reverse use dependence; APD is maximally increased at normal heart rates (increasing the risk of EAD-induced tachycardias), but during a tachycardia their desired effect declines. In fact, many of the newer selective IKr blockers have been shown to produce torsades de pointes arrhythmias in clinical trials (49). An ideal class III antiarrhythmic agent, rather than having reverse use dependent APD prolongation, should have little effect during normal sinus heart rates but steeply increase APD as the heart rate accelerates when tachycardia or fibrillation strikes. There seems to be two feasible ways to accomplish this. A first possibility is to develop a class III drug which increases APD by activating an inward $\mathrm{Na}^{+}$current as the cell depolarizes. In order to make such a drug rate-dependent (i.e., to increase APD proportionately to increases in heart rate), the drug would need to bind to its receptor during the upstroke of the action potential, slowly dissociate from the receptor during the plateau phase, and rapidly dissociate during the diastolic phase. Thus, as the heart rate increases, APD increases proportionately because of both a greater occupancy of the activator receptor (because of binding during the upstrokes which are increased as the heart rate increases) and a decreased dissociation from the activator receptor (because of slow dissociation during the plateaus which are increased, and fast dissociation during the diastolic intervals which are shortened as the heart rate increases) (50). The second possibility is to develop a rate-dependent class III antiarrhythmic drug that increases APD by blocking outward $\mathrm{K}^{+}$channels (50). The most promising target appears to be the slowly activating component of the delayed rectifier current (IKs). This current, as a result of incomplete deactivation of the channels, significantly increases in magnitude at rapid rates and accounts for the reverse rate dependence of selective IKr blockers (34). A selective blocker of IKs might therefore be a class III agent which is particularly effective at increased heart rates. Further research on these types of drugs may lead to the development of a rate-dependent class III antiarrhythmic agent which could effectively prevent arrhythmias and decrease mortality in treated patients. 


\section{REFERENCES}

1. Lucchesi BR, Chi L, Friedrichs GS, Black SC, Uprichard ACG. Antiarrhythmic versus antifibrillatory actions: inference from experimental studies. American Journal of Cardiology 72: 25F-44F; 1993.

2. Mitchell LB. Treatment of ventricular arrhythmias after recovery from myocardial infarction. Annual Review of Medicine 45: 119-38; 1994.

3. Stanton MS. Class I antiarrhythmic drugs: quinidine, procainamide, disopyramide, lidocaine, mexiletine, tocainamide, phenytoin, moricizine, flecainide, propafenone. In: Zipes DP, Jalife J, editors. Cardiac Electrophysiology: From Cell to Bedside. Philadelphia, PA: WB Saunders Company; 1995.

4. Kodama I, Honjo H, Kamiya K, Toyama J. Two types of sodium channel block by class I antiarrhythmic drugs studied by using Vmax of action potential in single ventricular myocytes. Journal of Molecular and Cellular Cardiology 22(1): 1-12; 1990.

5. Bigger JT, Hoffman BF. Antiarrhythmic drugs. In: Gilman AG, Rall TW, Nies AS, Taylor P, editors. Goodman and Gilman's the Pharmacological Basis of Therapeutics. New York, NY: McGraw Hill; 1990.

6. Salata JJ, Wasserstorm JA. Effects of quinidine on action potentials and ionic currents in isolated canine ventricular myocytes. Circulation Research 62: 324-337; 1988.

7. Kohlhardt M, Fichtner H, Frobe U, Herzig JW. On the mechanisms of drug-induced blockade of $\mathrm{Na}^{+}$ currents: interaction of antiarrhythmic compounds with DPI-modified single cardiac $\mathrm{Na}^{+}$channels. Circulation Research 64: 867-881; 1989.

8. Duan D, Fermini B, Nattel S. Potassium channel blocking properties of propafenone in rabbit atrial myocytes. Journal of Pharmacology and Experimental Therapeutics 264: 1113-1123; 1993.

9. Cardiac Arrhythmia Suppression Trial (CAST) investigators preliminary report: effect of encainide and flecainide on mortality in a randomised trial of arrhythmia suppression after myocardial infarction. New England Journal of Medicine 321: 406-412; 1989.

10. Campbell TJ. Subclassification of class I antiarrhythmic drugs: enhanced relevance after CAST. Cardiovascular Drugs and Therapy 6(5): 519-528; 1992.

11. Singh BN. Controlling cardiac arrhythmias: to delay conduction or to prolong refractoriness? Cardiovascular Drugs and Therapy 3: 671-674; 1990.

12. Wit AL, Coromilas J. Role of alterations in refractoriness and conduction in the genesis of reentrant arrhythmias: implications for antiarrhythmic effects of class III drugs. American Journal of Cardiology 72(16): 3F-12F; 1993.

13. Hohnloser SH. Indications and limitations of class II and III antiarrhythmic drugs in atrial fibrillation. Pace-Pacing \& Clinical Electrophysiology 17(5 Pt 2): 1019-1025; 1994.

14. Wong J, Feng J, Nattel S. Class III antiarrhythmic drug action in experimental atrial fibrillation: differences in reverse use dependence and effectiveness between d-sotalol and the new antiarrhythmic drug ambasilide. Circulation 90: 2032-2040; 1994.

15. Zanetti LA. Sotalol: a new class III antiarrhythmic agent. Clinical Pharmacy 12(12): 883-891; 1993. 
16. Hoffmeister HM, Beyer M, Seipel L. Hemodynamic effects of the D- and L-isomers of sotalol on normal myocardium. Cardiovascular Drugs and Therapy 5(6): 1027-1033; 1991.

17. Leatham EW, Holt DW, McKenna WJ. Class III antiarrhythmics in overdose. Presenting features and management principles. Drug Safety 9(6): 450-462; 1993.

18. Beyer T, Brachmann J, Kubler W. Comparative effects of d-sotalol and l-sotalol on the atrioventricular node of the rabbit heart. Journal of Cardiovascular Pharmacology 22(2): 240-246; 1993.

19. Mason JW on behalf of ESVEM investigators: A comparison of seven anti-arrhythmic drugs in patients with ventricular tachyarrhythmias. New England Journal of Medicine 329: 452-458; 1993.

20. Fitton A, Sorkin EM. Sotalol: an updated review of its pharmacological properties and therapeutic use in cardiac arrhythmias. Drugs 46(4): 678-719; 1993.

21. Waldo AL, Camm AJ, Deruyter H, et al. Survival with oral d-sotalol in patients with left ventricular dysfunction after myocardial infarction - rationale, design, and method (The SWORD trial). American Journal of Cardiology 75(15): 1023-1027; 1995.

22. Advani SV, Singh BN. Pharmacodynamic, pharmacokinetic and antiarrhythmic properties of d-sotalol, the dextro-isomer of sotalol. Drugs 49(5): 664-679; 1995.

23. Sasyniuk BI, Brunet S. Proarrhythmic effects of d-sotalol in rabbit ventricle associated with differential effects on endocardial cells at slow heart rates. Circulation 90: I-146; 1994.

24. Sasyniuk BI, Brunet S. Torsade de pointes induced by quinidine, d-sotalol and E-4031 in the isolated rabbit heart: importance of interval dependent dispersion of repolarization. PACE 18: 904; 1995.

25. Nattel S. Comparative mechanisms of action of antiarrhythmic drugs. American Journal of Cardiology 72: 13F-17F; 1993.

26. Kadish AH, Chen RF, Schmaltz S, Morady F. Magnitude and time course of beta-adrenergic antagonism during oral amiodarone therapy. Journal of the American College of Cardiology 16: 1240-1245; 1990.

27. Middlekauff HR, Wiener I, Stevenson WG. Low-dose amiodarone for atrial fibrillation. American Journal of Cardiology 72(16): 75F-81F; 1993.

28. Ceremuzynski L. Secondary prevention after myocardial infarction with class III antiarrhythmic drugs. American Journal Of Cardiology 72(16): 82F-86F; 1993.

29. Camm AJ, Julian D, Janse G, et al. on behalf of the EMIAT investigators. The European Myocardial Infarct Trial (EMIAT). American Journal of Cardiology 72: 95F-98F; 1993.

30. Sanguinetti MC: Modulation of potassium channels by antiarrhythmic and antihypertensive drugs. Hypertension 19: 228-236; 1992.

31. Spinelli W, Moubarak IF, Parsons RW, Colatsky TJ. Cellular electrophysiology of WAY-123,398, a new class III antiarrhythmic agent: specificity of IK block and lack of reverse use dependence in cat ventricular myocytes. Cardiovascular Research 27(9): 1580-1591; 1993.

32. Chadwick CC, Ezrin AM, O'Connor B, Volberg WA, Smith DI, et al. Identification of a specific radioligand for the cardiac rapidly activating delayed rectifier $\mathrm{K}^{+}$channel. Circulation Research 72(3): 707- 
$714 ; 1993$.

33. Martin CL, Chinn K. Contribution of delayed rectifier and inward rectifier to repolarization of the action potential: pharmacologic separation. Journal of Cardiovascular Pharmacology 19(5): 830-837; 1992.

34. Jurkiewicz NK, Sanguinetti MC. Rate-dependent prolongation of cardiac action potentials by a methanesufonanilide class III antiarrhythmic agent. Specific block of rapidly activating delayed rectifier K+ current by dofetilide. Circulation Research 72(1): 75-83; 1993.

35. Kiehn J, Villena P, Beyer T, Brachmann J. Differential effects of the new class III agent dofetilide on potassium currents in guinea pig cardiomyocytes. Journal of Cardiovascular Pharmacology 24(4): 566-572; 1994.

36. Sanguinetti MC, Jurkiewicz NK, Scott A, Siegl PKS. Isoproterenol antagonizes prolongation of refractory period by the Class III antiarrhythmic agent E-4031 in guinea pig myocytes: mechanism of action.

Circulation Research 68: 77-84; 1991.

37. Wallace AA, Stupienski RF, Brookes LM, Selnick HG, Claremon DA, Lynch JJ Jr. Cardiac electrophysiologic and inotropic actions of new and potent methanesulfonanilide class III antiarrhythmic agents in anesthetized dogs. Journal of Cardiovascular Pharmacology 18(5): 687-695; 1991.

38. Baskin EP, Serik CM, Wallace AA, et al. Effects of new and potent methanesulfonanilide class III antiarrhythmic agents on myocardial refractoriness and contractility in isolated cardiac muscle. Journal of Cardiovascular Pharmacology 18(3): 406-414; 1991.

39. Nabih MA, Prcevski P, Fromm BS, Lavine SJ, et al. Effect of ibutilide, a new class III agent, on sustained atrial fibrillation in a canine model of acute ischemia and myocardial dysfunction induced by microembolisation. Pace-Pacing and Clinical Electrophysiology 16(10): 1975-1983; 1993.

40. Buchanan LV, Turcotte UM, Kabell GG, Gibson JK. Antiarrhythmic and electrophysiologic effects of ibutilide in a chronic canine model of atrial flutter. Journal of Cardiovascular Pharmacology 22(1): 10-14; 1993.

41. Lee KS. Ibutilide, a new compound with potent class III antiarrhythmic activity, activates a slow inward $\mathrm{Na}^{+}$current in guinea pig ventricular cells. Journal of Pharmacology and Experimental Therapeutics 262(1): 99-108; 1992.

42. Lee KS, Tsai TD, Lee EW. Membrane activity of class III antiarrhythmic compounds; a comparison between ibutilide, d-sotalol, E-4031, sematilide and dofetilide. European Journal of Pharmacology 234(1): $43-53 ; 1993$.

43. Woosley RL. New antiarrhythmic drugs. In: Zipes DP, Jalife J, editors. Cardiac Electrophysiology: From Cell to Bedside. Philadelphia, PA: WB Saunders Company; 1995.

44. Buchanan LV, Kabell G, Brunden MN, Gibson JK. Comparative assessment of ibutilide, d-sotalol, clofium, E-4031, and UK-68,798 in a rabbit model of proarrhythmia. Journal of Cardiovascular Pharmacology 22(4): 540-549; 1993.

45. Busch AE, Malloy K, Groh WJ, Varnum MD, Adelman JP, Maylie J. The novel class III antiarrhythmics NE-10064 and NE-10133 inhibit IsK channels expressed in Xenopus oocytes and IKs in guinea pig cardiac myocytes. Biochemical and Biophysical Research Communications 202(1): 265-270; 1994. 
46. Fermini B, Jurkiewicz NK, Jow B, et al. Use-dependent effects of the class III antiarrhythmic agent NE10064 (azimilide) on cardiac repolarization - block of delayed rectifier potassium and l-type calcium currents. Journal of Cardiovascular Pharmacology 26(2): 259-271; 1995.

47. Bril A, Faivre JF, Forest MC, et al. Electrophysiological effect of BRL-32872, a novel antiarrhythmic agent with potassium and calcium channel blocking properties, in guinea pig cardiac isolated preparations. Journal of Pharmacology and Experimental Therapeutics 273(3): 1264-1272; 1995.

48. Gout B, Nichols AJ, Feuerstein GZ, Bril A. Antifibrillatory effects of BRL-32872 in anesthetized Yucatan minipigs with regional myocardial ischemia. Journal of Cardiovascular Pharmacology 26(4): 636-644; 1995.

49. Sasyniuk BI, Carmeliet E. Role of potassium channel modulators in the treatment of cardiac arrhythmias. In: Cuello AC, Collier B, editors. Pharmacological Sciences: Perspectives for Research and Therapy in the Late 1990s. Basel: Birkhauser; 1995.

50. Hondeghem LM. Development of class III antiarrhythmic agents. Journal of Cardiovascular Pharmacology 20(Suppl. 2): S17-S22; 1992.

\section{BIOGRAPHY}

Videl Essebag is currently a third-year medical student at McGill University (Montreal, Quebec). His research on antiarrhythic drugs was conducted at McGill University during his second year of medical education.

Copyright (C) 1995 by MJM 